\title{
Computing measures of simplicity of fit for loadings in factor-analytically derived scales
}

\author{
JAMES S. FLEMING \\ Embry-Riddle Aeronautical University, Prescott, Arizona
}

\begin{abstract}
A very simple structure is sought when factor analysis is used to develop measurement scales. The SIMLOAD program computes measures of factorial simplicity for rows and columns of loading matrices (usually the factor pattern) as well as some overall measures. These include Kaiser's (1974) index of factorial simplicity for variables (rows), the author's scale fit index for factors (columns), Bentler's (1977) scale-free matrix measure, and hyperplane counts. Routine use of these measures is recommended for multifactor scale development. The measures may also be useful in more general factor applications and in confirmatory as well as exploratory analyses. SIMLOAD also computes factor scale intercorrelations, scale alpha coefficients (including alpha when an item is removed), and sorted loadings for ease of interpretation.
\end{abstract}

How does one evaluate "goodness" of structure in exploratory factor analysis when the test item writer strives for a very simple structure in the sense described below? Thurstone (1947) provided a number of general criteria for simple structure of loadings, but item writers often wish to construct "item-pure" tests that exhibit what is sometimes called simple cluster structure. In such cases, the ideal factor-loading matrix ${ }^{1}$ takes the following form (after rows and columns of loadings are appropriately arranged):

$$
\begin{array}{lllll}
\mathrm{X} & 0 & 0 & \ldots & 0 \\
\mathrm{X} & 0 & 0 & \ldots & 0 \\
\mathrm{X} & 0 & 0 & \ldots & 0 \\
. & & & \\
. & & & & \\
0 & \mathrm{X} & 0 & \ldots & 0 \\
0 & \mathrm{X} & 0 & \ldots & 0 \\
. & & & & \\
. & & & & \\
0 & 0 & 0 & \ldots \mathrm{X} \\
0 & 0 & 0 & \ldots \mathrm{X},
\end{array}
$$

wherein each variable has a nonzero loading on one and only one factor. In this configuration, an $\mathrm{X}$ indicates a salient (large) loading. Although it is possible to achieve such a pattern in confirmatory factor analysis, it is next to impossible in exploratory factor analysis, since very small loadings, which might be statistically insignificant, will still differ from zero by small amounts due to random error. Thus, the example above represents for the test item writer a kind of ideal target in which each variable saturates one and only one factor, and items that contribute to complexity (i.e., those that load on more than one factor) are candidates for elimination.

Correspondence concerning this article and software and reprint requests should be addressed to J. Fleming, 2275 Pine Dr., Prescott, AZ 86305 (e-mail: flemingjs@earthlink.net).
A number of measures can be used to gauge the goodness of a solution in terms of its approximation to this ideal fit, on the basis of simplicity of rows (i.e., the extent to which just one variable saturates a given factor), columns (i.e., the degree of separation of salient from nonsalient loadings), and total matrix. These measures can supplement other (unifactorial) scale development measures in selecting or refining items in multifactor scales (see, e.g., DeVellis, 1991, for methods of selecting items for unifactor scales). Such an application is illustrated subsequently, in the first of two data examples. However, the measures presented here may also be of interest in assessing the complexity of factor solutions in more general applications of factor analysis, per the second data example. Although the measures were developed within the context of exploratory factor analysis, they may also be applied to confirmatory analysis. The SIMLOAD computer program computes all of the measures discussed in this article.

\section{Row Simplicity}

Kaiser (1974) presented an index of factorial simplicity $\left(\mathrm{IFS}_{i}\right)$, which is computed for each $i$ th variable. It ranges from 0 (but values below .50 are unacceptable) to 1.0 (when all but one of the loadings are zero). The measure is based on the quartimax criterion (Carroll, 1953; Saunders, 1953; Wrigley \& Neuhaus, 1954). A total IFS is also computed. Let $a_{i j}$ be an element of factor pattern matrix A with $m$ rows (variables) and $p$ columns (factors). Then, for the $i$ th variable

$$
\mathrm{IFS}_{i}^{2}=\frac{p\left[\sum_{k} a_{i k}^{4}-\left(\sum_{k} a_{i k}^{2}\right)^{2}\right]}{\left[(p-1)\left(\sum_{k} a_{i k}^{2}\right)^{2}\right]} .
$$


Kaiser (1974) believed that IFS values of .80 or more were meritorious.

\section{Column Simplicity}

A measure newly proposed here to assess the simplicity of each factor (i.e., column), called the factor scale fit index $\left(\mathrm{SFI}_{j}\right)$ for each of the factors, is defined as

$$
\mathrm{SFI}_{j}=1-\operatorname{SSQ}\left(\mathrm{N}_{j}\right) / \mathrm{SSQ}\left(\mathrm{T}_{j}\right)
$$

where SSQ stands for sum of squares. Thus, $\operatorname{SSQ}\left(\mathrm{N}_{j}\right)$ is the sum of squares of the nonsalient (small) loadings and $\operatorname{SSQ}\left(\mathrm{T}_{j}\right)$ is the sum of squares of total loadings, both salient and nonsalient, for column $\mathrm{j}$. This measure also ranges from (a theoretical) 0 to 1.0 , the perfect 1.0 being achieved when all nonsalient loadings are zero. Actually, a value of .50 means that salient items are no better than nonsalient items in defining scales; practically speaking, values of at least .80 are desirable, as is the case with the Kaiser index.

A total measure is also computed using a formula similar to the one presented above, except that the second term on the right consists of the ratio of sums of squares of nonsalient to total loadings for all columns (i.e., for the total matrix).

The computation of $\mathrm{SFI}_{j}$ requires some means of discriminating salient from nonsalient loadings. One method of achieving this is to predefine a SIMLOAD value (i.e., a LOVAL parameter) for loadings presumed to be in the hyperplane (e.g., .15). Alternatively, the discrimination may be made on a more relative basis, with the cutoff values being determined according to the criterion of Kaiser and Cerny (1978), discussed in the next section. For sorting purposes, a HIVAL parameter is also entered for loadings that are particularly salient, such as .45. Thus, items above HIVAL on one factor but below LOVAL on all others are good candidates for retention. In keeping with an item analysis approach, an item cannot be assigned to more than one factor scale (if more than one item exceeds HIVAL, the program still assigns that variable only to the factor with the highest loading for that variable for sorting purposes).

$\mathrm{SFI}_{j}$ is similar to a measure I proposed a number of years ago (Fleming, 1985), but I believe the present measure is better rationalized. ${ }^{2}$ Let $\mathrm{SSQ}(\mathrm{Sj})$ be the sum of squares for the salient loadings for the $j$ th factor. The quantity $\mathrm{SSQ}(\mathrm{Sj}) / \mathrm{SSQ}(\mathrm{Nj})$ is a signal-to-noise ratio which will be large to the extent that the nonsalient loadings are small relative to the salient ones. However, it is undefined in the ideal case (in which all nonsalient loadings are zero). $\mathrm{SFI}_{j}$ is a measure derived from this concept, but it is 1.0 in the limiting case that all nonsalient loadings are zero. As such, it is similar in form and logic to coefficient alpha (Cronbach, 1951).

\section{Matrix (Overall) Simplicity}

SIMLOAD also computes a measure introduced by Bentler (1977) that ranges from 0 (very complex struc- ture) to 1.0 (very simple structure). The measure-a kind of generalized variance-is defined as

$$
\mathrm{s}=\left|\mathrm{D}^{-\frac{1}{2}} \mathrm{~B}^{\prime} \mathrm{B} \mathrm{D}^{-\frac{1}{2}}\right|,
$$

where $\mathrm{B}$ is a matrix of squared factor loadings and $\mathrm{D}=$ Diag ( $\left.\mathrm{B}^{\prime} \mathrm{B}\right)$. If the loading matrix is of the form presented above as the ideal, the matrix product presented above will be an identity matrix with determinant 1.0. Bentler proved that this is a scale-free measure (i.e., it is not affected by arbitrary scaling of the columns). He also described orthogonal and oblique rotations (called orthosim and $o b$ lisim, respectively) to maximize this criterion.

Loadings in the hyperplane (i.e., essentially zero except for random error) can also be counted as a measure of adequacy in approximating the simple cluster structure. For $m$ variables and $p$ factors, $m(p-1)$ is the ideal hyperplane count. ${ }^{3}$ In exploratory analysis, the standard error of factor loadings is difficult to approximate, so rules of thumb for identifying factors that are statistically insignificant are often employed rather than statistical criteria. For this purpose, SIMLOAD computes counts of items that are less than LOVAL in absolute value (e.g., .15) and also computes hyperplane counts according to a criterion rationalized by Kaiser and Cerny (1978) on the basis of an absolute power function of the loadings (Kendall \& Stuart, 1969). For each factor,

$$
f_{j}=\left[\frac{\sum_{i}\left(a_{i j}^{2}\right)^{\frac{1}{b}}}{m}\right]^{\frac{b}{2}} .
$$

Kaiser and Cerny (1978) chose $b=4$ to give the best results using somewhat subjective criteria (although this can be adjusted in SIMLOAD). Loadings of less than $f_{j}$, then, are considered to be in the hyperplane. This function adjusts for the relative size of loadings on each factor: The criterion is larger for factors with large loadings and smaller for factors with relatively small loadings. (Increasing $b$ makes $f_{j}$ smaller, and decreasing $b$ makes $f_{j}$ larger.)

Rather than assigning a specific numeric value for LOVAL (as was discussed in the previous section), an alternative low relative value may be determined by the Kaiser-Cerny procedure, separately for each factor. In this case, near-zero values for sorted loadings will differ for each factor, depending on the relative magnitudes of the loadings.

\section{Further Analyses of Factor Scale}

To further facilitate the work of the test item writer, SIMLOAD also computes coefficient alpha on the basis of the items comprising each factor scale, whether standardized or raw. It also computes alpha values for each scale when each item is removed. These statistics are optional and can be computed only if the correlation matrix and standard deviations (or covariance matrix) are input as 
well as the loadings. Also, it is assumed that the loading matrix is a factor pattern matrix in standardized metric (i.e., it is not arbitrarily scaled).

In addition, the program computes an intercorrelation matrix for factor scales (which is not the same as factor or factor score correlation matrices) and prints a sorted loading matrix in which variables and factors are ordered to resemble the ideal matrix displayed at the beginning of this article. For ease of reading and interpretation, sorted loadings below LOVAL are set to zero and factor definers are starred.

\section{The Value of Simplicity Measures as General Tools}

Although I have described this program as a useful tool in the test developer's kit, the measures presented here may be useful for other kinds of data as well. This is true of Example 2, in which the subtests of the WISC-R (Wechsler, 1974) were analyzed (as opposed to the analysis at the item level). This example was chosen because of its familiarity to most psychologists. Another potential application is in the comparison of the effectiveness of various rotations in producing such a simple cluster structure.

This program was designed for applications of exploratory factor analysis. However, the measures provided can also be useful in confirmatory factor analysis, except that, in the latter case, hyperplane counts should be based on absolute zero loadings rather than on an arbitrary value for small loadings (i.e., LOVAL should be set to 0 ).

\section{Examples}

Example 1: Scale of Parents' Beliefs About Children's Health (DeVellis, 1991). This scale illustrates the behavior of the measures in a scale development application that is, in fact, a textbook example of simple cluster structure, as can be seen, possibly with minor exceptions, in Table 1 . The analysis is based on 396 parents' ratings of their beliefs concerning their children's health.

Several of the variables presented in Table 1 exceed the often suggested arbitrary value of .15 for cutting off small from large values. However, this is an orthogonal (varimax) solution; an even simpler structure would be almost guaranteed with an oblique rotation. Thus, to analyze these data, I chose to use the (usually more liberal) KaiserCerny criterion for hyperplane values, which, as has been noted, is a function of the relative size of the low coefficients in comparison with the high values for computing the scale fit indices. With this criterion, the cutoff hyperplane values for the two factors were .38 and .30 . Thus, as can be seen in Table 1, all IFS values are above .85, and the SFI values are at least .95 . The Bentler measure is .995. The hyperplane count of 12 is also equal to the ideal count. No items are candidates for elimination, as was also suggested by the author's own analysis based on the factor structure, alpha coefficients, and changes in the values of alphas if items are removed (DeVellis, 1991). The scale alphas are both approximately .80 .

Example 2: WISC-R factors. Table 2 shows factor pattern coefficients from the analysis of $11 \mathrm{WISC}-\mathrm{R}$ scales (Weschler, 1974) for a sample of 211 learning-disabled children with three obliquely rotated (direct oblimin) factors (i.e., verbal, performance, and freedom from distractibility). The data were originally analyzed by Tabachnick and Fleming (1986). Also shown are the measures of simplicity.

Although this is not a test construction application (in that scales rather than items are being evaluated), it is still interesting to see where the complexities exist in the interpretation of the WISC-R factors and to observe the behavior of the various measures when two of the three fac-

Table 1

Factor Analysis of Parental Belief Items Concerning Children's Health

\begin{tabular}{lrrr}
\hline & \multicolumn{3}{c}{ Factors } \\
\cline { 2 - 4 } Items & $\begin{array}{c}\text { Parental } \\
\text { Influence }\end{array}$ & $\begin{array}{c}\text { Luck or } \\
\text { Chance }\end{array}$ & IFS \\
\hline 11. I can do a lot to help my child stay well. & .786 & -.221 & .856 \\
9. I can do a lot to help my child stay strong and healthy. & .748 & -.185 & .879 \\
4. I can do a lot to prevent my child from getting hurt. & .719 & -.023 & .998 \\
5. I can do a lot to prevent my child from getting sick. & .659 & -.158 & .889 \\
7. The things I do at home with my child are an important & & & \\
part of my child's well-being. & .658 & .019 & .998 \\
1. I have the ability to influence my child's well-being. & .597 & -.141 & .897 \\
8. My child's safety depends on me. & .519 & -.074 & .964 \\
6. Whether my child avoids sickness is just a matter of luck. & -.092 & .822 & .976 \\
10. My child's health is largely a matter of good fortune. & -.109 & .786 & .962 \\
3. Luck plays a big part in determining how healthy my child is. & -.078 & .754 & .978 \\
12. Whether my child stays healthy or gets sick is just a matter of luck. & -.173 & .738 & .900 \\
2. Whether my child avoids injury is just a matter of luck. & -.116 & .636 & .932 \\
SFI & .979 & .954 & \\
\hline
\end{tabular}

Note-IFS, index of factorial simplicity; SFI, scale fit index. From Scale Development: Theory and Applications (p. 104), by R. F. DeVellis, 1991, Newbury Park, CA: Sage. Copyright 1991 by Sage Publications. Reprinted with permission. 
Table 2

WISC-R Factor Pattern Matrix for a Sample of LearningDisabled Children, With Associated Measures of Simplicity

\begin{tabular}{lrrrr}
\hline \multirow{2}{*}{\multicolumn{1}{c}{ Subtests }} & \multicolumn{4}{c}{ Factors } \\
\cline { 2 - 5 } & \multicolumn{1}{c}{ VC } & \multicolumn{1}{c}{ PO } & \multicolumn{1}{c}{ FD } & IFS \\
\hline 1. Information & 0.840 & -0.020 & -0.110 & .974 \\
2. Similarities & 0.720 & 0.180 & -0.280 & .739 \\
3. Arithmetic & 0.670 & -0.020 & 0.240 & .834 \\
4. Vocabulary & 0.840 & 0.000 & -0.070 & .990 \\
5. Comprehension & 0.740 & 0.140 & -0.050 & .942 \\
6. Digit span & 0.550 & -0.070 & 0.410 & .543 \\
7. Picture completion & 0.080 & 0.720 & -0.230 & .849 \\
8. Picture arrangement & 0.020 & 0.560 & 0.170 & .875 \\
9. Block design & 0.120 & 0.690 & 0.160 & .884 \\
10. Object assembly & -0.060 & 0.810 & 0.000 & .992 \\
11. Coding & -0.080 & 0.150 & 0.880 & .946 \\
SFI & .990 & .961 & .642 & \\
\hline
\end{tabular}

Notes-VC, verbal comprehension; PO, perceptual orientation; FD, freedom from distractibility; IFS, index of factorial simplicity; SFI, scale fit index.

tors exhibit rather simple structures but the third is more complex; hence, the SFI is unacceptably low for the latter factor. All values of Kaiser's (1974) IFS are at least meritorious ( $>.80)$ for all but two subtests, but the IFS value for digit span, which saturates two factors, is especially low. The overall IFS is .903, the overall SFI is .917, and the overall Bentler measure is high at .977 . Thus, although the overall measures appear acceptable, the individual values of IFS and SFI suggest room for improvement in the structure. (If these were items rather than subtests, removal of the offending items would seem justified.) The hyperplane counts are as follows: For the .15 criterion, 15 values $(45.5 \%)$ are in the hyperplane; for the Kaiser-Cerny criterion, $18(54.5 \%)$ are in the hyperplane; and the ideal count is $22(66.7 \%)$. Standardized alphas for the factors of verbal comprehension, perceptual orientation, and freedom from distractibility are $.821, .687$, and 0.0 , respectively (since only the coding subscale defines the third factor scale); raw alphas are very similar. If digit span is removed, the alpha for verbal comprehension increases only a bit, to .837 .

Conclusions and recommendation. The examples presented here illustrate the behavior of the measures of pattern simplicity in typical applications of both scale development and more general analysis. They also give a feel of how the measures perform in practice. The overall measures (IFS, SFI, and Bentler statistic), when considered alone, can all be somewhat misleading: they can be large even when there is still room for improvement, which is detectable only by more careful inspection of the individual measures. It is recommended that the objective measures discussed here be routinely employed in multidimensional scale development applications.

\section{Program Description}

Language and operating system. SIMLOAD was written in Compaq Visual Fortran 6.5.0 and compiled under Windows 98. SIMLOAD is an interactive program that runs on later versions of Windows.
Input. Input of basic parameters is interactive. Matrix and (optional) factor and variable labels are input from a user-named file. Interactive input includes a title, floatingpoint parameters HIVAL (criterion for salient loadingse.g., .35) and LOVAL (criterion for loading in the hyperplane-e.g., .15), and specification of processing options (e.g., is coefficient alpha desired?). As an alternative to specifying a single low value, these may be determined separately for each factor using the Kaiser-Cerny hyperplane criterion. A correlation matrix and standard deviations (or a covariance matrix) must also be included on the input file if alpha coefficients for factor scales are desired.

Output. Output is stored on to a user-named file. It includes the measures of row simplicity due to Kaiser (1974), column (factor scale) simplicity (the present article), and matrix simplicity (Bentler's, 1977, index, and hyperplane counts as described above). In addition, alpha coefficients for factor scales may also be requested as a further aid to the test constructor, along with scale alphas as recalculated with each item removed in turn. The program also prints original and sorted loadings. In the latter case, loadings in the hyperplane are printed as zeros; those above HIVAL are starred for ease of interpretation. As a further aid in interpretation, the matrix of sorted loadings should be visually compared with the ideal matrix shown above.

Limitations. The program accepts up to 200 variables and 30 factors.

Documentation. User documentation is provided with the program (including test data as well as sample input and output).

Availability. The SIMLOAD runtime package, user documentation, sample test data, and Fortran subroutines for performing the calculations discussed here are available from the author at no cost. Include a blank floppy disk and a SASE with sufficient postage. Alternatively, you may send your e-mail address to flemingjs@ earthlink.net if you would like a zipped copy of the program and sample documentation e-mailed directly to you.

\section{REFERENCES}

BARRETT, P. (1996). An evaluation of the psychometric properties of the concept 5.2 Occupational Personality Questionnaire. Journal of Occupational \& Organizational Psychology, 69, 1-19.

BENTLER, P. M. (1977). Factor simplicity index and transformations. Psychometrika, 42, 277-295.

CARroll, J. B. (1953). An analytical solution for approximating simple structure in factor analysis. Psychometrika, 18, 23-38.

CronbaCH, L. J. (1951). Coefficient alpha and the internal structure of tests. Psychometrika, 16, 297-334.

DE VELlis, R. F. (1991). Scale development: Theory and applications. Newbury Park, CA: Sage.

FLEMING, J. S. (1985). An index of fit for factor scales. Educational \& Psychological Measurement, 45, 725-728.

Harman, H. H. (1976). Modernfactor analysis (3rd. ed.). Chicago: University of Chicago Press.

KAISER, H. F. (1974). An index of factorial simplicity. Psychometrika, 39, 31-35

KAiser, H. F., \& CERnY, B. A. (1978). Casey's method for fitting hyperplanes from an intermediate orthomax criterion. Multivariate Behavioral Research, 13, 395-401.

KENDALl, M. G., \& STUART, A. (1969). The advanced theory of statistics (3rd. ed., Vol. 1). New York: Hafner. 
SAUNDERS, D. R. (1953). An analytic method for rotation to orthogonal simple structure [Abstract]. American Psychologist, 8, 428.

TABACHNICK, B. G., \& Fleming, J. S. (1986, April). WISC-R profile and factor analyses of learning disabled subgroups. Paper presented at the annual meeting of the Western Psychological Association, Seattle.

Thompson, B., \& DANiEL, L. G. (1996). Factor analytic evidence for the construct validity of scores: An historical overview and some guidelines. Educational \& Psychological Measurement, 45, 203-209.

Thurstone, L. L. (1947). Multiple factor analysis. Chicago: University of Chicago Press.

WECHSLER, D. (1974). Manual for the Wechsler Intelligence Scale for Children-Revised. San Antonio, TX: Psychological Corporation.

Wrigley, C., \& Neuhaus, J. O. (1954). The quartimax method: An analytic approach to orthogonal simple structure. British Journal of Statistical Psychology, 7, 81-91.

\section{NOTES}

1. The terms loading and factor-loading matrix are notoriously ambiguous (Harman, 1976; Thompson \& Daniel, 1996). In the orthogonal case, the pattern matrix (of regression weights) and the structure matrix (of correlations between latent and manifest variables) are identical. In oblique analysis, there are many loading matrices: primary pattern, primary structure, reference pattern (almost never discussed), and reference structure (preferred by Thurstone, 1947, for interpretation of factors). The primary pattern matrix and the reference structure matrix are generally the simplest in structure (but note that the reference structure matrix is proportional to the primary pattern matrix); hence, I recommend interpreting them for item analysis when attempting to approximate the ideal loading matrix. The primary structure matrix, though a useful adjunct in factor interpretation in its own right, can never be made as simple or as close to ideal in form as these other loading matrices. Thus, in this article, loadings may refer either to primary pattern or reference structure in oblique analysis or to the pattern/structure matrix in the orthogonal case; but note that if alpha coefficients are desired, a pattern matrix in the oblique case is assumed when my SIMLOAD program is used.

2. Barrett (1996) has defined a measure similar to my earlier index of fit for factor scales that uses absolute loadings rather than squared loadings.

3. Hyperplane counts can sometimes be misleading as an index of simple cluster structure. For example, if all high loadings are as predicted except two, in which one variable loads on two factors and the other fails to load on any factor, the ideal count is still reached. As always, interpretation must be tempered by common sense.

(Manuscript received September 24, 2002; revision accepted for publication May 28, 2003.) 